\title{
The Water-Amorphous Calcium Carbonate Interface and Its Interactions with Amino Acids
}

\author{
R. Innocenti Malini, ${ }^{\dagger} \dagger^{\dagger} \ddagger \odot$ A. R. Finney, ${ }^{\dagger}$ S. A. Hall, ${ }^{\S}$ C. L. Freeman, ${ }^{\dagger}$ and J. H. Harding ${ }^{\dagger}$ \\ ${ }^{\dagger}$ Department of Materials Science and Engineering, University of Sheffield, Mappin Street, Sheffield S1 3JD, U.K. \\ ${ }^{\ddagger}$ Laboratory for Biomimetic Membranes and Textiles, Empa, Swiss Federal Laboratories for Materials Science and Technology, St. \\ Gallen 9014, Switzerland \\ ${ }^{\S}$ National Research Council Canada, Ottawa, Ontario K1A 0R6, Canada
}

\section{Supporting Information}

ABSTRACT: Amorphous calcium carbonate is often the first phase to precipitate from solution during the mineralization of calcium carbonate, before the formation of one of the crystalline polymorphs. In vivo, this phase is believed to be essential for the manufacture of minerals displaying nonequilibrium morphologies. The precipitation of this, usually transient, phase and its subsequent transformation into one of the crystalline polymorphs can be controlled by organic molecules. Here, we present a series of molecular dynamics simulations that explore the amorphous calcium carbonate-water interface, the attachment of amino acids onto both hydrous and anhydrous amorphous calcium carbonate, and their effect on the surface. The results show that surface ions have a different coordination number distribution from bulk ions and can diffuse up to two orders of magnitude faster than their bulk counterparts, suggesting that crystallization is much more likely to occur in this region. All the amino acids investigated bind to the amorphous calcium carbonate surfaces. However, acidic amino acids have a clear preference for the surface of amorphous

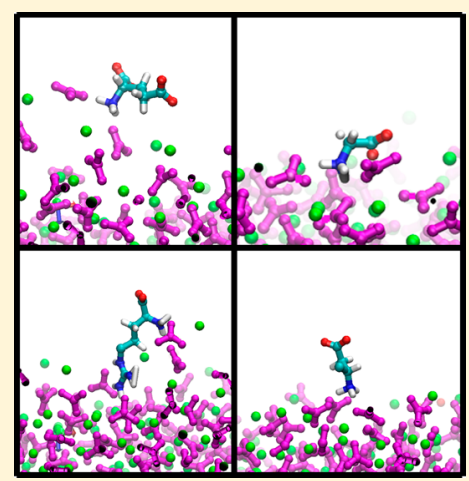
$\mathrm{CaCO}_{3} \cdot \mathrm{H}_{2} \mathrm{O}$. The favored mode of interaction of the amino acids is through amine and/or guanidine moieties. The important ramifications of the results for our understanding of protein-mineral interactions are discussed.

\section{INTRODUCTION}

Living organisms have the ability to precipitate minerals- the growth of which is controlled from the bottom-up-with morphologies and properties finely tuned for their task. While this capability is affected by several factors, two seem to be particularly important: the initial precipitation of an amorphous precursor $^{1}$ and the interactions between the mineral phase and organic molecules. ${ }^{2}$ Nucleation of precursor amorphous phases occurs throughout the animal kingdom. ${ }^{3,4}$ Thus, understanding the binding and the effect of organic molecules on the structure of this intermediate phase and on its potential energy landscape is important for understanding biomineralization. The results could then be used to design new manufacturing processes for advanced materials. ${ }^{5}$

Interactions between amorphous calcium carbonate (ACC) and organic molecules seem to be essential in the formation of the required crystalline polymorph. ${ }^{1,4,6}$ While studying molluscs, both Falini et al. ${ }^{7}$ and Belcher et al. ${ }^{8}$ suggested that polymorph selection can be controlled by macromolecules associated with both the prismatic and nacre layers of mollusc shells. Later, when an amorphous precipitate was first observed in vivo, ${ }^{9,10}$ it was suggested that macromolecules influenced polymorph selection by modifying the structure of the amorphous precursor. Further support for this idea was obtained when the amorphous precipitate in both aragonite-forming molluscs ${ }^{11}$ and calciteforming sea urchins ${ }^{12}$ showed structural patterns that were different and matched the relevant final polymorph.
The stabilization of an amorphous phase is extremely important in some living organisms. First of all, it is an easily accessible feedstock for the supply of ions to a growing crystal, and second, it can be essential for regulating both the location and the time frame of crystallization, ${ }^{4}$ which needs to be tightly controlled to ensure the proper functioning of the final material. Experimental investigations have shown that, in vivo, the amorphous precursor is in contact with proteins containing substantial numbers of glutamic acid and glycine residues. ${ }^{13}$ In vitro experiments showed that polyaspartic acid was able to delay crystallization in solution and stabilize liquid-like amorphous aggregates (PILP - polymer-induced liquid precursors) in both calcium carbonate and calcium phosphate solutions. ${ }^{5,14-16}$

Not only are acidic domains of proteins and peptides able to interact with the precipitating mineral phase, but also basic residues can have an impact on nucleation and growth. In a recent study, Cantaert et al. showed that the formation of PILP observed with polyaspartic acid in solution ${ }^{5}$ could be replicated using a basic amino acid: poly(allylamine hydrochloride), ${ }^{17}$ indicating that both positively and negatively charged molecules can have an impact on nucleation and growth. In another study, Tavafoghi and Cerruti analyzed the effect of glutamic acid and arginine on the precipitation of hydroxyapatite (HAP) from

Received: June 21, 2017

Revised: September 14, 2017

Published: October 3, 2017 
solution. ${ }^{18}$ Arginine was better at delaying the precipitation of $\mathrm{HAP},{ }^{18}$ and additionally, its incorporation within the final crystal was more pronounced than glutamic acid. ${ }^{18}$ The results obtained by Tavafoghi and Cerruti also showed that nucleation and growth of mineral phases in solution can be modified by amino acids. ${ }^{18}$ Amino acids are cheaper and easier to synthesize than complex proteins and may prove to be more viable for industrial applications.

Experimentally, it is not yet possible to follow the molecular mechanism leading to specific observations, such as those discussed above (even though recent advances in technology is allowing the observation of yet smaller length scales). These mechanisms can, however, be investigated using molecular simulations. Freeman et al. used molecular dynamics (MD) simulations to understand how ovocleidin-17 (a protein found in avian eggshell) binds to ACC (and calcite) and its effect on the free energy landscape of calcium carbonate nanoparticles. ${ }^{19-22}$ The simulation showed that the binding of ovocleidin-17 to an amorphous surface occurred mainly through arginine residues in the random coil of the protein. ${ }^{22}$ Raiteri et al. used a modified version of the CHARMM force field (for the organic species) to analyze the interaction of calcium and carbonate ions (modeled using a potential specifically derived for the simulation of calcium carbonate $^{23}$ ), their ion pairs, amorphous nanoparticles, and the (10.4) calcite surfaces with citrate, aspartate, and acetate. ${ }^{24}$ The simulations showed that the free energy of binding of acetate and aspartate to an amorphous surface was very similar, suggesting an interaction mainly via the carboxylate group. In another study, Saharay and Kirkpatrick used metadynamics to obtain the free energy curve of the interaction of functional group assays representing different amino acids. ${ }^{25}$ The functional groups were modeled using guanidine, acetate, acetic acid, and ethanol. As the strongest binding energies were found for the guanidine and acetate groups, it was suggested that these acted as nucleation sites for the crystallization of calcium carbonate minerals. ${ }^{25}$

The binding of organic molecules to ion pairs is considerably different from surface binding, as shown by Raiteri et al. ${ }^{24}$ The surface can form water layers, which could impede the binding, or modify it. ${ }^{26}$ Raiteri et al. ${ }^{24}$ only considered acidic additives with different numbers of carboxylate groups and did not consider positively charged functional groups in their study; thus, it is desirable to analyze the role of charge-charge interactions in additive-ACC binding in a self-consistent study.

Here, we investigate the binding of glutamic acid, aspartic acid, glycine, and arginine to both hydrous and anhydrous ACC surfaces using classical MD simulations. The protonation state of the molecules was selected to represent the most likely states in a solution at $\mathrm{pH}$ 7.4: two of the amino acids are negatively charged (glutamic acid and aspartic acid), one is positively charged (arginine) and the fourth is net neutral (glycine). MD was chosen to obtain an atomistic view of surface adsorption and associated mechanisms at the atomic scale. The results show that all amino acids bind to the hydrous and anhydrous ACC surfaces. The favored mode of interaction observed was hydrogen bonding between either the guanidine or amine groups. Additionally, we investigated the water-ACC interface, showing that this is relatively wide with a structure that evolves during the timescale of the simulations, and that the ions in that region diffuse up to two orders of magnitude faster than the bulk ions. The implications of the results for biomineralization are discussed.

\section{METHODS}

System Preparation. The ACC slabs were prepared using previously described methods, ${ }^{27}$ which lead to a structure with atomic pair distribution functions and ionic coordination numbers that compare well with experimental data. ${ }^{28}$ Briefly, the ions were randomly packed in a cubic cell using the program PACKMOL (the minimum distance between molecules was set to $2.2 \AA) .{ }^{29}$ Two different bulk ACC configurations were prepared: one contained 294 formula units of $\mathrm{CaCO}_{3}\left(\mathrm{ACC}_{1}\right)$ and the second 294 formula units of $\mathrm{CaCO}_{3} \cdot \mathrm{H}_{2} \mathrm{O}$ $\left(\mathrm{ACC}_{2}\right)$. The configurations were then melted, as bulk materials, for $1 \mathrm{~ns}$ at $3000 \mathrm{~K}$ using a time step of $1 \mathrm{fs}$ in an NVT ensemble using DL POLY classic version 1.9. ${ }^{30}$ A Nosé-Hoover thermostat with a 0.1 ps relaxation time was used to control the temperature of the simulation. Using the final configuration and the same simulation input parameters, the system was subsequently cooled to $300 \mathrm{~K}$ in steps of $300 \mathrm{~K}$. The ensemble was then changed to NPT using a Nosé-Hoover barostat to control the pressure of the simulation (1.0 ps relaxation time), set at 1 atm. MD was performed until the configurational energy, volume, and temperature converged. At the end of this equilibration, $\mathrm{ACC}_{1}$ and $\mathrm{ACC}_{2}$ simulation cells were cubic boxes with lengths of $26.03 \AA$ and $27.84 \AA$, respectively. The statistical data presented were extracted from $5 \mathrm{~ns}$ of additional simulation time following equilibration.

To prepare the solution-ACC configurations, PACKMOL was used again. ${ }^{29}$ The minimum distance between the different molecules in the system was set to $2.2 \AA$ as before. The distance between the amino acids and the ACC surface was set to $5.0 \AA$. For charged amino acids, a counterion was inserted in the simulation box, either $\mathrm{Cl}^{-}$(for arginine) or $\mathrm{Na}^{+}$(for glutamic acid and aspartic acid) to ensure that the sum of atomic charges in the simulation was zero. Both $\mathrm{ACC}_{1}$ and $\mathrm{ACC}_{2}$ surfaces were solvated using 2050 water molecules, creating ACC slabs separated by water. The total dimension (ACC slab + water) of the $\mathrm{ACC}_{1}$ slab system was $26.03 \AA \times 26.03 \AA \times 112.84 \AA$, while for $\mathrm{ACC}_{2}$ the final dimensions were $27.84 \AA \times 27.84 \AA \times 104.42 \AA$.

Simulation Details. For all the following work, we used the force field developed by Raiteri and Gale $(2010)^{23}$ to model the ACC. As suggested in the specification of the carbonate force field, water molecules were treated using the SPC/Fw model. ${ }^{31}$ The amino acid intramolecular interactions were modeled using the standard AMBER force field set (ff12SB), ${ }^{32,33}$ which has been used in a large number of previous analyses of the interaction between biomoleules and the calcium carbonate system, allowing for a consistent comparison of results. ${ }^{22,34,35}$ Intermolecular interactions between the organic and mineral phase were fitted using the Schröder method described by Freeman et al. $^{36}$ The intermolecular interactions of the organic molecules with water were obtained by using the standard LorentzBerthelot rules. A verification of the intermolecular parameters obtained was performed by investigating the free energy of binding between $\mathrm{Ca}^{2+}$ ions and aspartic acid for which experimental values have been reported: $-6.7 \mathrm{~kJ} \mathrm{~mol}^{-1}$. $^{37}$ After obtaining the free energy curve, the entropy dependence was corrected following the method detailed by Raiteri et al. to allow comparison with their results. ${ }^{24}$ The curve obtained is presented in Figure S1. The change in the free energy found was $-4.5 \mathrm{~kJ}$ $\mathrm{mol}^{-1}$. This value is less favorable than the experimental binding energy but is closer than previous values obtained via simulation $(-12.2 \mathrm{~kJ}$ $\mathrm{mol}^{-1}$ and $\left.-16.7 \mathrm{~kJ} \mathrm{~mol}^{-1}\right) .^{24,38}$

Four different configurations of each amino acid, chosen randomly, were simulated close to both a hydrous and anhydrous ACC slab. Initially, the amino acids were tethered using a harmonic potential $(k=$ $2.5 \mathrm{~kJ} \mathrm{~mol}^{-1} \AA^{-2}$ ) at a distance of approximately $5 \AA$ from the surface. All of the simulations were initially relaxed in a NVT ensemble implemented using a Nosé-Hoover thermostat (relaxation time of $0.1 \mathrm{ps}$ ). The time step was set at $1 \mathrm{fs}$, and the simulation was performed for 10 ns. During this equilibration, the configurational energy continuously decreased, which was probably due to a slow remodelling of the ionic network. The ensemble was therefore set to NPT using a Nosé-Hoover thermostat and barostat (relaxation time of 0.1 and $1 \mathrm{ps}$, respectively) in order to reach a steady state with respect to the configurational energy. Most simulations were continued for approximately $30 \mathrm{~ns}$, during which the variation of the configurational energy 

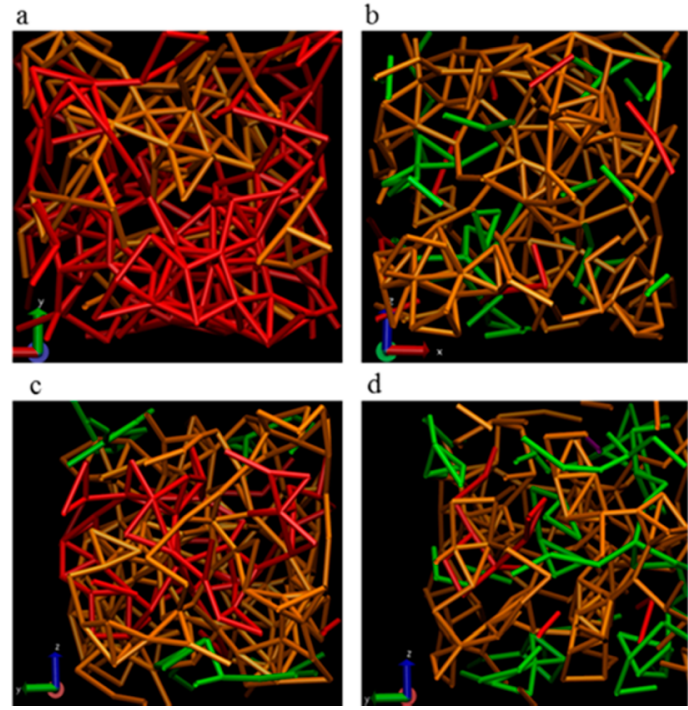

d

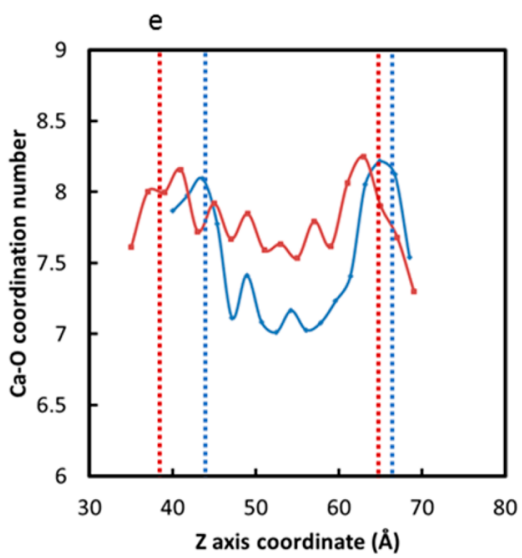

Figure 1. Representation of $\mathrm{Ca}^{2+}$ ions with different average coordination numbers in the different systems analyzed. The $\mathrm{Ca}^{2+}$ ions with an oxygen coordination number between 6 and $7\left(\mathrm{Ca}_{6}\right)$ are in red, the ones with an oxygen coordination number between 7 and $8\left(\mathrm{Ca}_{7}\right)$ are shown in orange and with an oxygen coordination number between 8 and $9\left(\mathrm{Ca}_{8}\right)$ are shown in green. The $\mathrm{Ca}_{x}-\mathrm{Ca}_{x}$ interactions are shown using a line representation where the cutoff between like calciums is set at $5.2 \AA$, which is the first minimum in the Ca-Ca radial distribution function. Side views are shown of (a) bulk $\mathrm{ACC}_{1}$, (b) bulk $\mathrm{ACC}_{2}$, (c) slab $\mathrm{ACC}_{1}$, and (d) slab $\mathrm{ACC}_{2}$. In (c) and (d) the interfaces with the solution are at the top and bottom of the images along the $Z$ direction, shown in the image by the blue arrow. Also shown are the $\mathrm{Ca}-\mathrm{O}$ coordination values as a function of proximity to the ACC slab surfaces (e) with the $\mathrm{ACC}_{1}$ (blue) and $\mathrm{ACC}_{2}$ (red). The vertical lines represent the position where the density of the ions is half that of the bulk structure. We consider this as a transition point between the solution and solid ACC.

decreased; however, no complete convergence in the energy was found during our simulations. The configurations characterized by the lowest configurational energy were then simulated without the additional tether for an additional $15 \mathrm{~ns}$. The results presented are from the last $5 \mathrm{~ns}$ of these simulations.

For all the above simulations, the electrostatic interactions were treated using the Ewald summation method and the cutoff for the intermolecular interactions was set to $9 \AA$. Additionally, in all the simulations two $\mathrm{Ca}^{2+}$ ions in the center of the slabs were fixed to their positions to avoid any drift of the system both in the MD and the umbrella sampling simulations presented below.

Free Energy of Binding. Umbrella sampling was used to calculate the Helmholtz free energy of binding by using PLUMED 1.3, a plug-in that can be used in combination with different $\mathrm{MD}$ packages. ${ }^{39}$ For these calculations, ACC slabs equilibrated as described above but without amino acids were used to ensure that the restructuring in the presence of an amino acid during the long time scale simulation did not have an impact on the results. The order parameter of the simulations was the position of the carbon attached to the amine group of the amino acid, which was restrained at different values of the vector normal to the ACC plane. Initially, the absolute position was varied by $1 \AA$ in each window until a distance of $15 \AA$ from the surface was reached. For each window, an initial run of 200 ps was performed to optimize the molecule's position. Subsequently, each window was simulated for at least 3 ns. If the free energy changes were observed to have sizable error bars (calculated using the Monte Carlo bootstrap error analysis), the time was extended by either 1 or $2 \mathrm{~ns}$, until convergence was reached (the error was below room temperature energy). If the free energy curve obtained did not converge with time or the error was still large, additional windows were added in the regions where barriers were found (the distributions of the distances sampled for all the umbrella sampling windows are presented in Figure S2). The free energies for the reactions were then obtained using the weighted histogram analysis method (WHAM), a code developed by Grossfield. ${ }^{40}$ For data presentation, the free energy curves were displaced so that the minima were all at the same distance from the surface. This facilitates comparison, as the ACC surface is rough (see Figure S3) and the amino acids interacted with different regions on the surface.
Analysis of Average Time of Water Molecules within Ionic Coordination Shells. The average times for water molecules occupying the first coordination shell of carbonate and calcium ions were calculated by counting the number of consecutive time steps that a water molecule stayed within the first coordination shell (determined as the first minimum in the RDF) of the element of interest. If a water molecule left its coordination shell for more than 2 ps then the count would be reset. To avoid spurious effects arising from water molecules transiting close to the ions without entering within their coordination shell, in the averages presented water molecules that resided within the coordination shell for less than 3 ps were not counted. These effects can arise because in the first minimum of the RDF the value is not zero, so molecules could enter within the threshold distance without actually entering within the coordination sphere of the ion of interest.

It is important to emphasize that the measured value will be different from the often quoted residence times. For the latter, the probability distribution of a water molecule remaining for at least a time $t+\Delta t$ is calculated and then fitted with an exponential function, from which the residence time is obtained. To be able to do this accurately, however, an average is usually taken over ions/atoms in positions that are symmetrically equivalent. ${ }^{41}$ In the case of ACC this is not possible as the surface is rough and evolves dynamically; thus, the distributions obtained were too noisy to be fitted accurately.

To ensure that our force field could reproduce residence times in the literature, we performed a short simulation including one calcium ion in 3000 water molecules for $5 \mathrm{~ns}$. The residence time calculated was $0.17 \mathrm{~ns}$ which is comparable to, but slightly smaller than, the value quoted by De La Pierre et al., ${ }^{41} \sim 0.2$ ns. This difference is probably due to the short simulation time used.

For a surface, the residence time does not give a measure of the time it takes for a water molecule to transition from a bound state to bulk water. Instead, it measures the time that a water molecule resides in the first coordination shell of the ion of interest. A water molecule could jump from one ionic site to the other, without passing through the bulk solution when leaving the previous coordination shell. Unlike the Pavese et al. ${ }^{42}$ model, previously used extensively for the simulation of calcium carbonate surfaces, the force field model of Raiteri and Gale ${ }^{43}$ shows some ion dissolution. ${ }^{44}$ Thus, the ACC surface is rough (as mentioned above and shown in Figure S3), and, as it will be shown, water molecules 

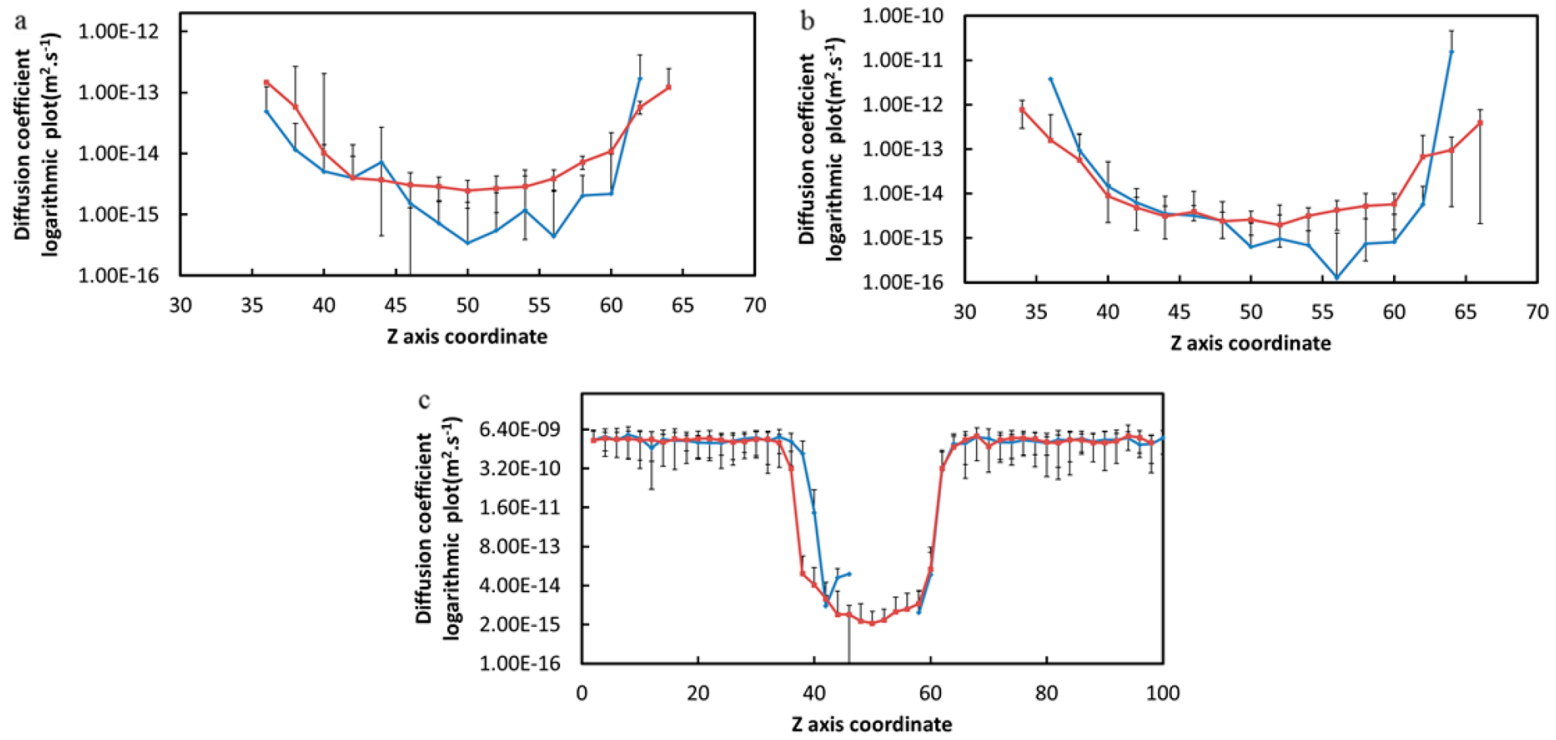

Figure 2. Diffusion profiles of the different elements of the system along the $\mathrm{Z}$ axis. (a) $\mathrm{Ca}^{2+}$, (b) the carbon in the $\mathrm{CO}_{3}{ }^{2-}$ ion, and (c) the oxygen of the water molecule. The blue line depicts the result obtained from $\mathrm{ACC}_{1}$ and the red line from $\mathrm{ACC}_{2}$. The ACC in all figures resides approximately between 37 and $63 \AA$ along the $Z$ axis coordinate. In (c), the gap in the blue curve is due to the absence of water molecules in the center of the $\mathrm{ACC}_{1}$ slab. The error bars represent the standard deviation of the averaged data. (Note that some error bars are larger than what would be expected in the bulk and close to the interface. This is because we used the initial position of the ions when computing the averages as a function of the position normal to the surface and sometimes the ion diffused to a position where its dynamics were very different from its starting point.)

penetrate extensively inside it during equilibration. The residence time of the water molecules will be highly dependent upon their depth within the structure and their interactions with the ionic framework. Some water molecules which are embedded within the ionic structure will have infinite residence times.

\section{RESULTS}

ACC Bulk and Surface Structure. The PDFs obtained for both $\mathrm{ACC}_{1}$ and $\mathrm{ACC}_{2}$ were approximately the same (Figure S4) and compared well to experimental data, ${ }^{28}$ confirming that structural water does not significantly affect ion coordination spheres in ACC, as recently shown by Bushuev et al. ${ }^{45}$

Analysis of the average number of oxygens (both from water molecules and carbonate ions) in $\mathrm{Ca}^{2+}$ ion coordination shells showed a broad distribution spanning values between 6 and 9 , as shown in previous work. ${ }^{27}$ This was then analyzed by separating the $\mathrm{Ca}^{2+}$ ions as a function of the average number of oxygens in their coordination shell. The results showed that most of the $\mathrm{Ca}^{2+}$ ions in bulk $\mathrm{ACC}_{1}$ had either an average coordination number between 6 and $7\left(\mathrm{Ca}_{6}\right)$ or between 7 and $8\left(\mathrm{Ca}_{7}\right)$, as shown in Figure 1a. While the distribution of average coordination shows a single, broad peak in the bulk structure (see for example Figure S5), it is interesting to note that the spatial distribution of $\mathrm{Ca}^{2+}$ with relatively low $\left(\mathrm{Ca}_{6}\right)$ or high $\left(\mathrm{Ca}_{7}\right)$ coordination is nonuniform. Two distinct regions are shown using red and orange bonds in Figure 1a. In $\mathrm{ACC}_{2}$ the structure was dominated by $\mathrm{Ca}_{7}$ species with a small number of $\mathrm{Ca}_{8}$ (oxygen coordination number between 8 and 9) and $\mathrm{Ca}_{6}$. While the number of the latter two species was small, their clustering into dimers, trimers, and (rarely) larger clusters could still be observed within the bulk structure.

The results obtained for both $\mathrm{ACC}_{1}$ and $\mathrm{ACC}_{2}$ suggest that, in a bulk macroscale scenario, different ACC domains could exist and that these would be populated by calcium ions with different coordination numbers. This could be linked to the reported ACC polyamorphism. ${ }^{41}$
When water molecules were added to the ACC in the simulation cell to create an interfacial system, the structures of both hydrous and anhydrous ACC reconfigured compared to their bulk counterparts. In $\mathrm{ACC}_{1}$, shown in Figure $1 \mathrm{c}$, more $\mathrm{Ca}_{7}$ species were observed at the interface than the bulk, while the $\mathrm{Ca}_{6}$ were concentrated at the center of the slab. Additionally, $\mathrm{Ca}_{8}$ formed at the surface. This observation was confirmed by plotting the distribution of coordination number as a function of absolute position, presented in Figure 1e. In the center of the $\mathrm{ACC}_{1}$ slab, the average coordination number is close to seven, and as we move toward the interface this increases to eight. The decrease of the average, after this maximum close to the interface, is due to the dissolution of $\mathrm{Ca}^{2+}$ ions into solution, which, as Raiteri et al. showed, have an equilibrium coordination number close to 7.2 when using the current force field. ${ }^{46}$ This effect was much less pronounced in $\mathrm{ACC}_{2}$ (Figure 1d) with small increases in the number of $\mathrm{Ca}_{8}$ species at the surface, while the number of both $\mathrm{Ca}_{6}$ and $\mathrm{Ca}_{7}$ species slightly decreased. $\mathrm{ACC}_{2}$ was, nonetheless, still dominated by $\mathrm{Ca}_{7}$. The average coordination number slightly increases as you go from the center of the slab to the interface (Figure 1e), confirming that as the number of water molecules increases within ACC, the number of oxygens in the $\mathrm{Ca}^{2+}$ ions coordination shell increases. This is interesting as within anhydrous ACC and in water the number of oxygens within the coordination shell of $\mathrm{Ca}^{2+}$ ions is closer to 7 (7.2 in water ${ }^{43}$ and 7.0 in $\mathrm{ACC}_{1}$ ), whereas the number gets closer to 8 as the amount of water within ACC increases. Thus, water molecules, being less bulky than carbonate ions, increase the number of oxygens in the $\mathrm{Ca}^{2+}$ ion coordination shells, within amorphous calcium carbonate, by penetrating into the spaces left between the carbonates.

Between the water and the ACC, regardless of the hydration level, a diffuse interface formed. In the case of $\mathrm{ACC}_{1}$, which was anhydrous, Figure $\mathrm{S} 6$ shows that water penetrated approximately $6 \AA$ into the structure. Figure $S 6$ also shows that, compared to the crystalline surfaces, ${ }^{22,47}$ the ACC surfaces do not structure water 


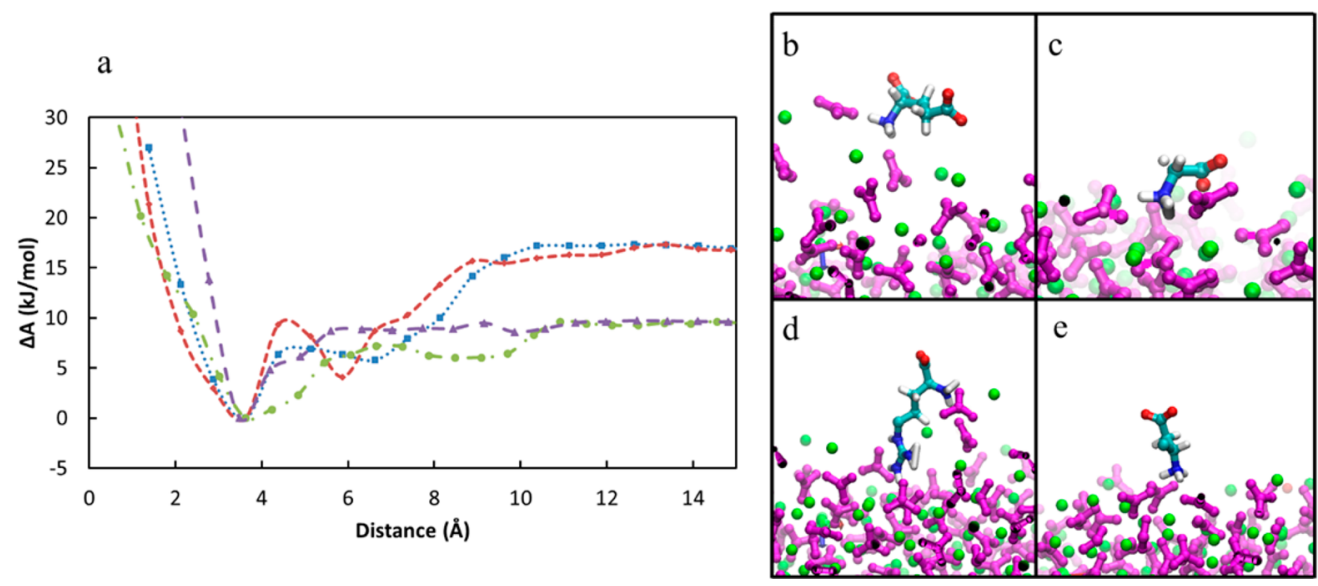

Figure 3. (a) Binding free energies of amino acids to the $\mathrm{ACC}_{1}$ surface as a function of the distance between the surface and the carbon attached to the amine. The green curve was obtained for glutamic acid, the purple one for aspartic acid, the red curve depicts glycine, while the blue one was obtained for arginine. Amino acids on the surface of $\mathrm{ACC}_{1}$. (b) Glutamic acid, (c) glycine, (d) arginine, and (e) aspartic acid. Water was removed from the images for clarity.

molecules as strongly. The water layers are diffuse and do not differ much from the bulk structure. This is in contrast to the water structure at the interface with crystalline surfaces. ${ }^{44,47,48}$ In the case of calcite, for example, the first water layer can be four times as dense as in the bulk liquid. ${ }^{22}$ By contrast, here the first peak is at most 1.2 times the bulk value (Figure S6). This is important as incoming molecules will not have to compete with water molecules strongly bound to the surface as seen for crystal surfaces. A lack of regularly arranged water molecules implies that the free energy barrier associated with the binding process will be smaller than that for a crystalline surface. ${ }^{23}$

The diffusion coefficients of the water molecules and the ions along the $Z$ direction (perpendicular to the surface formed between the water molecules and the ACC) of the system have also been analyzed and are presented in Figure 2

Within a bulk solid, the movement of the atoms can be separated into several mechanisms: vibration around lattice positions, short-range hopping from one site to another, and long-range bulk diffusion. It is possible that with longer time scale simulations a long-range diffusion could be captured; however, this would have little effect on the overall bulk structure. In this work we present the short distance diffusion coefficients (i.e., capturing the first two mechanisms). The values measured in this way encompass short-range hopping between sites and help us to understand the dynamics and mobility of the ions in the structure. The mobility of the ions is likely to be important in understanding relaxations around binding molecules and structural relaxations. Additionally, this methodology has been employed for other simulation work on ACC allowing us to compare and contrast. The values compare well with previous ACC simulations (Bushuev et al. ${ }^{45}$ ) and are larger than values obtained for calcite, ${ }^{49}$ which is sensible.

Whereas the diffusion of water molecules at the surface does not appear to be strongly perturbed compared to the bulk, there was an increase of up to two orders of magnitude in the diffusion coefficients of the surface ions compared to those in bulk ACC. An increased mobility suggests that ions could restructure around incoming molecules and that formation of crystalline nuclei or dissolution is potentially more likely. Interestingly, there is not a large difference in the diffusion coefficient of the ions between $\mathrm{ACC}_{1}$ and $\mathrm{ACC}_{2}$, suggesting that the presence of water within the bulk of the structure does not have an important effect on the dynamics of the system at the surface or in the bulk.

Binding of Amino Acids onto ACC. While the free energy profiles for the binding of amino acids approaching ACC surfaces have been calculated, and were observed to converge on the time scale of the simulations (at least $3 \mathrm{~ns}$ for each window for a total of $72 \mathrm{~ns}$ for the umbrella sampling simulations), the ACCs analyzed in this study were not fully relaxed, and the natural dynamics of ions in ACC were allowed to evolve during the simulations. Hence, there may be some reconstruction of the surface in different umbrella sampling windows that should reflect the natural restructuring of the ionic network as molecules approach the surface. Additionally, as shown in Figure S3, the surface is rough, with some ions being partially dissolved.

During the standard MD long-time scale simulations, it was observed that the configurational energy of the ACCs, shown in Figure S7, did not reach a steady state due to a very slow relaxation of the ionic network. This is similar to what was observed for nanoparticles by Bano et al. ${ }^{44}$ It is also important to realize that the shift in configurational energy observed is relatively small. As summarized in Table S1, it ranges between 1 and $-40 \mathrm{~kJ} \mathrm{~mol}^{-1} \mathrm{~ns}^{-1}$, which could match, for instance, the change in energy associated with the formation of a hydrogen bond each ns. A thorough analysis of the ACC structures using multiple order parameters including the Steinhardt $Q_{6}$ bond order parameter (Figures S8 and S9), the $\mathrm{Ca}-\mathrm{O}$ coordination number (Figure S10), the angles of the carbonates with respect to the $x-y$ plane (parallel to the surface) (Figures S11 and S12) and the radial distribution functions (Figure S13) did not indicate large variations in the different systems analyzed, and these differences could not be correlated with the different changes in configurational energies observed for the ACC. It is thus unlikely that the free energy of binding would be changed significantly by small reorganizations of the ACC surface like those associated with the observed downward shift in the total configurational energy. This was checked in detail for aspartic acid on anhydrous ACC. The umbrella sampling simulations were continued for $6 \mathrm{~ns}$, and the free energy obtained from the first half and the second half of the simulation time are presented in Figure S14a. In Figure S14b the change in configurational energy in one of the umbrella sampling window is shown. While the latter continued to decrease, the free energy changes 
obtained between the unbound and bound state for the two cases agree to within $1 \mathrm{~kJ} \mathrm{~mol}^{-1}$, and along the curves in Figure S14a, the difference in free energy changes are minimal (i.e., within the thermal energy available at room temperature). The free energies of binding calculated for all of the amino acids on both $\mathrm{ACC}_{1}$ and $\mathrm{ACC}_{2}$ are favorable, and their values lie between -18 and $-9 \mathrm{~kJ}$ $\mathrm{mol}^{-1}$

All of the free energy curves obtained for $\mathrm{ACC}_{1}$ are shown in Figure $3 \mathrm{a}$, and they all present a similar behavior. After the minimum, found close to the surface (bound state), there is a general increase in energy followed by a plateau (solvent-shared bound state). The absence of a transition barrier in the free energy curve between the two states is most likely due to the lack of structuring of the water molecules at the surface. As described above, in contrast to crystalline surfaces, ACC leads to only small changes in the density of water molecules at the surface (Figure S3). The plateau, instead, arises from interaction with partially dissolved ions. As the ACC surface is not flat but is rough and fluctuating, (Figure S3) the amino acids can interact with these ions as they transition from/to the bulk solution. After this plateau, the energy increases again slightly as the molecules enter the bulk solution. The only amino acid showing different behavior is glycine. Here there is a transition barrier of $10 \mathrm{~kJ}$ $\mathrm{mol}^{-1}$ between the bound state and the solvent shared bound state, which is $4 \mathrm{~kJ} \mathrm{~mol}^{-1}$ less stable than the bound state. This could be due to a slightly different configuration of the ions in the region where this molecule binds. Indeed, by looking at Figure $3 \mathrm{c}$, it can be seen that glycine seems more embedded in the surface than the other molecules. As none of the other free energy curves show any transition barrier, their attachment should be limited only by concentration, diffusion, and competition between molecules and other species in solution.

On $\mathrm{ACC}_{1}$ there is a clear difference between negatively charged and neutral or positively charged molecules. Arginine and glycine show binding energies of $-17 \mathrm{~kJ} \mathrm{~mol}^{-1}$, while aspartic acid and glutamic acid binding energies are close to -10 $\mathrm{kJ} \mathrm{mol}^{-1}$ (see Table 1). As presented in Figure $3 \mathrm{~b}-\mathrm{e}$, all of the

Table 1. Summary of the Free Energy of Binding $\left(\mathrm{kJ} \mathrm{mol}^{-1}\right)$

\begin{tabular}{lrc} 
& \multicolumn{1}{c}{$\mathrm{ACC}_{1}$} & $\mathrm{ACC}_{2}$ \\
arginine & $-17.4 \pm 0.5$ & $-15.4 \pm 0.6$ \\
aspartic acid & $-10.8 \pm 1.3$ & $-17.9 \pm 0.3$ \\
glutamic acid & $-9.4 \pm 0.4$ & $-13.2 \pm 0.2$ \\
glycine & $-16.8 \pm 0.2$ & $-17.9 \pm 0.4$ \\
\hline
\end{tabular}

amino acids bind to $\mathrm{ACC}_{1}$ via the amine group, or in the case of arginine, via the guanidine group. This is also observed in the density profiles obtained from the long time-scale simulations shown in Figure 4. The carboxylate groups only rarely interact with the surfaces. In contrast, the $\mathrm{H}_{\mathrm{N}}$ (hydrogens attached to nitrogen) density profile shown in red in Figure 4 tends to be closely followed by carbonates (purple line); thus, the free energy difference observed above could be due, mostly, to Coulombic energy effects.

$\mathrm{ACC}_{2}$ presents more variations between the free energy curves (Figure 5a). While the one obtained for glutamic acid is similar to what was described above, the results for the other curves are different. Glycine shows a broad minimum in the free energy, which slowly increases until the dissolved state is seen at large separations. Similarly, the free energy curve for aspartic acid does not display a metastable, solvent-shared bound state and the energy quickly increases with distance until dissolution. Arginine on $\mathrm{ACC}_{2}$ shows a very wide basin close to the surface with three distinct minima representing slightly different configurations shown in Figure S15. From Figure S15a, at the free energy minimum closest to the surface the arginine binds through all of the functional groups present in the molecule (which lie close to the surface). Figure S15b shows the most stable configuration, where the molecules bind only through the amine group. The final minimum, the furthest from the surface, arises from the interaction between the guanidine group and a carbonate ion (Figure S15c). Then, the free energy slightly increases to a small plateau and increases again as the molecule detaches from the surface. The binding free energy of the molecules on $\mathrm{ACC}_{2}$, ranges between -13.2 and $-17.8 \mathrm{~kJ} \mathrm{~mol}^{-1}$ (summarized in Table 1). In general, the binding free energy of the molecules on $\mathrm{ACC}_{2}$ is more favorable than $\mathrm{ACC}_{1}$. While for glycine and arginine the change between $\mathrm{ACC}_{1}$ and $\mathrm{ACC}_{2}$ is on the order of the room temperature thermal energy (about $2.5 \mathrm{~kJ} \mathrm{~mol}^{-1}$ ), aspartic acid attachment on $\mathrm{ACC}_{2}$ is $7.1 \mathrm{~kJ} \mathrm{~mol}^{-1}$ more favorable than on $\mathrm{ACC}_{1}$. This molecule is seen, in Figure 5e, to bind to the surface through both the carboxylate and amine groups, while on $\mathrm{ACC}_{1}$ the aspartic acid only binds via the amine group (Figure $3 \mathrm{e}$ ). This subtle change in the favored configuration could explain the change in energy.

As shown in Figure $5 b-e$, the binding on this surface also occurs mostly through the amine or guanidine groups. Aspartic acid and arginine, however, also bind via the carboxylate moiety. This is similar to what was observed in the density profiles of the long time scale simulations shown in Figure 6. The difference is that, in the density profiles in Figure 6, only glutamic acid binds via a carboxylate group. This again shows that different binding pathways are present on the surface and that these go through some continuous reconfiguration, at least on the time scale of the current simulations. This can have an effect on the way that the molecule binds to the surface. However, as for $\mathrm{ACC}_{1}$, there is a clear trend: the nitrogen-bearing groups are obviously the preferred mode of binding.

The density profiles presented in Figures 4 and 6 also show that, during long time scale simulations, some of the molecules slowly start to be incorporated within the structure. Often, the increase in density of the amines is closely followed by an increase in density of the carbonate ions. The surface, being more mobile than the bulk, reacts to the presence of the amino acid by optimizing the position of the ions. This allows a maximum interaction between the $\mathrm{O}_{\text {carbonate }}$ and the $\mathrm{H}_{\mathrm{N}}$ of the amine or guanidine groups.

The geometrical parameters of the hydrogen bonds formed between the amine groups and the carbonate ions are summarized in Table S2. In general, the donor-acceptor $\left(\mathrm{N}_{\text {amine }}-\mathrm{O}_{\text {carbonate }}\right)$ distance lies between 2.6 and $3.5 \AA$, and the angle formed $\left(\mathrm{N}_{\text {amine }}-\mathrm{H}_{\text {amine }}-\mathrm{O}_{\text {carbonate }}\right)$ lies between 144 and $171^{\circ}$. This agrees well with the gas phase geometry optimization at the B3LYP/6-311+G level of theory calculated in a previous investigation. ${ }^{25}$ The values depict hydrogen bonds that are mostly electrostatic.

Water Structure at ACC Surfaces and Effect of Amino Acid Binding. As recognized by Dorvee and Veis, the residence time of water molecules in coordination shells around the ions is an important consideration for the kinetics of nucleation, growth and crystallization. ${ }^{50} \mathrm{~A}$ decrease in the water residence time would probably indicate that the energy barrier at the transition between water molecules in ion coordination shells is smaller, and thus that exchange reactions will be facilitated. Organic molecules are known to modify water structure in their 

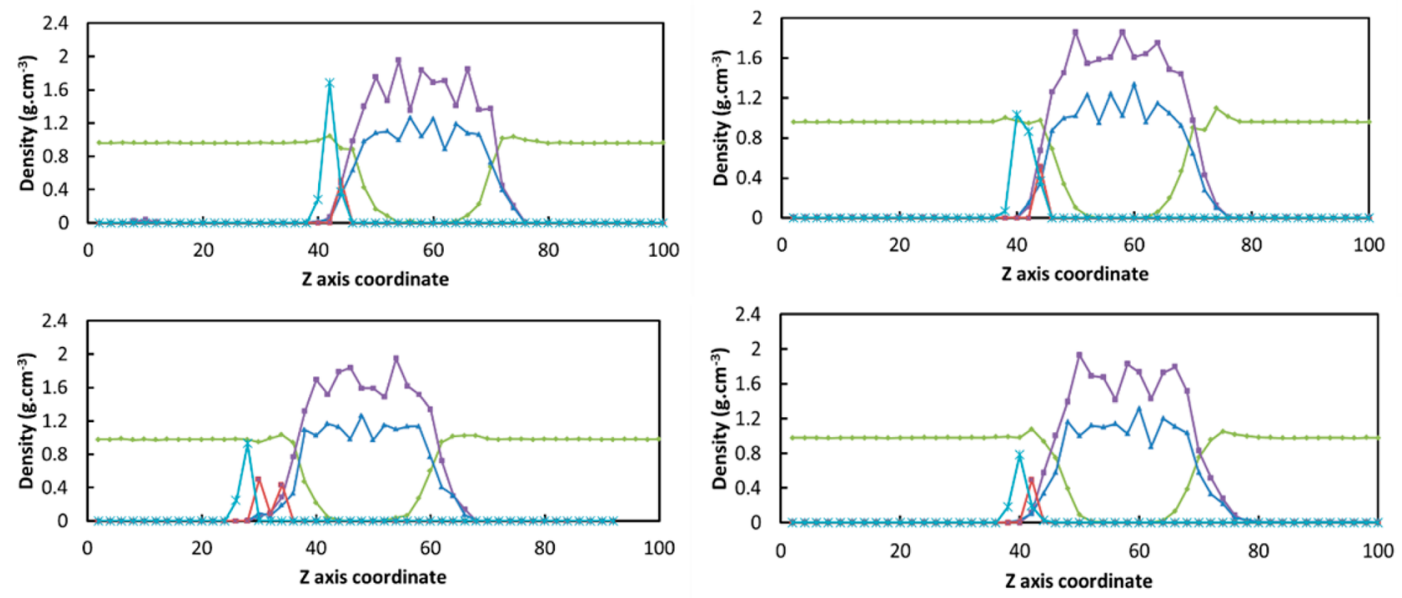

Figure 4. Density profiles of water (green line), carbonate (purple line), calcium (blue line), $\mathrm{N}$ (nitrogen in amine or guanidine group - red line), and carboxylate oxygens (light blue line), obtained from $\mathrm{ACC}_{1}$. From left to right and top to bottom: aspartic acid, glutamic acid, arginine, and glycine. The density of the carboxylate oxygens and the nitrogen of the amines was multiplied by 30 to make them visible in the image.
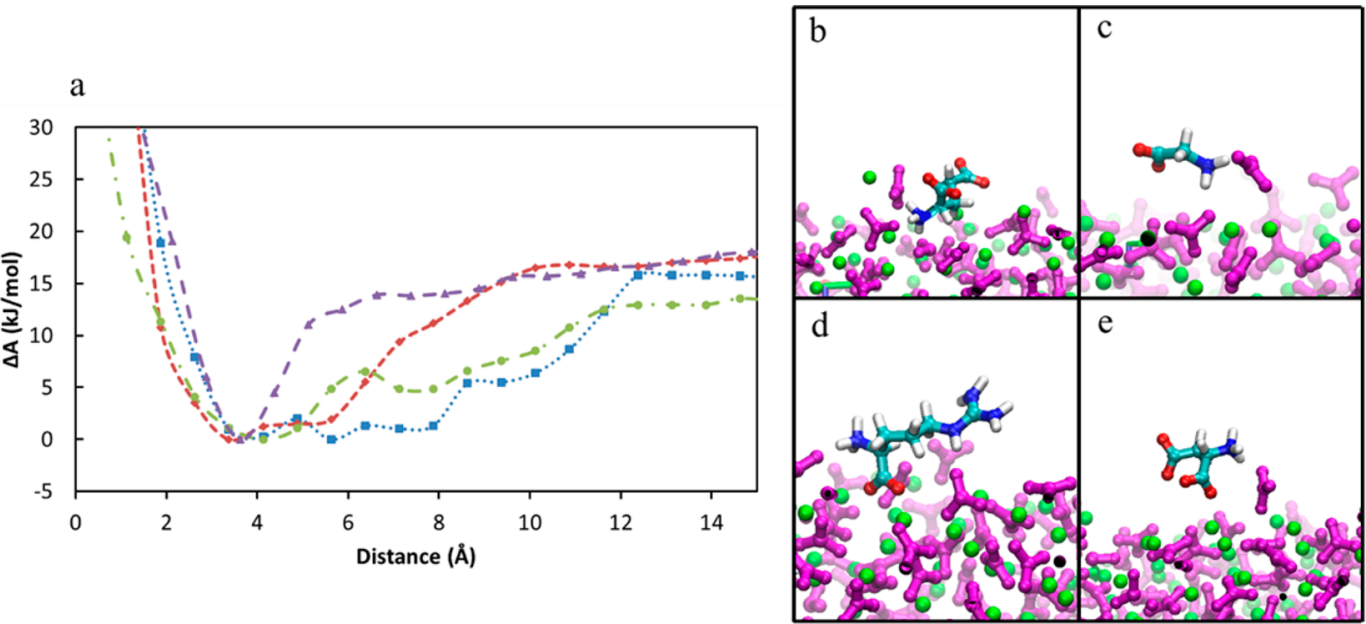

Figure 5. (a) Binding free energies of amino acids to an $\mathrm{ACC}_{2}$ surface as a function of the distance between the surface and the carbon attached to the amine. The green curve was obtained for glutamic acid, the purple one for aspartic acid, the red curve depicts glycine, while the blue one was obtained for arginine. Amino acids on the surface of $\mathrm{ACC}_{2}$. (b) Glutamic acid, (c) glycine, (d) arginine, and (e) aspartic acid. Water was removed from the images for clarity.
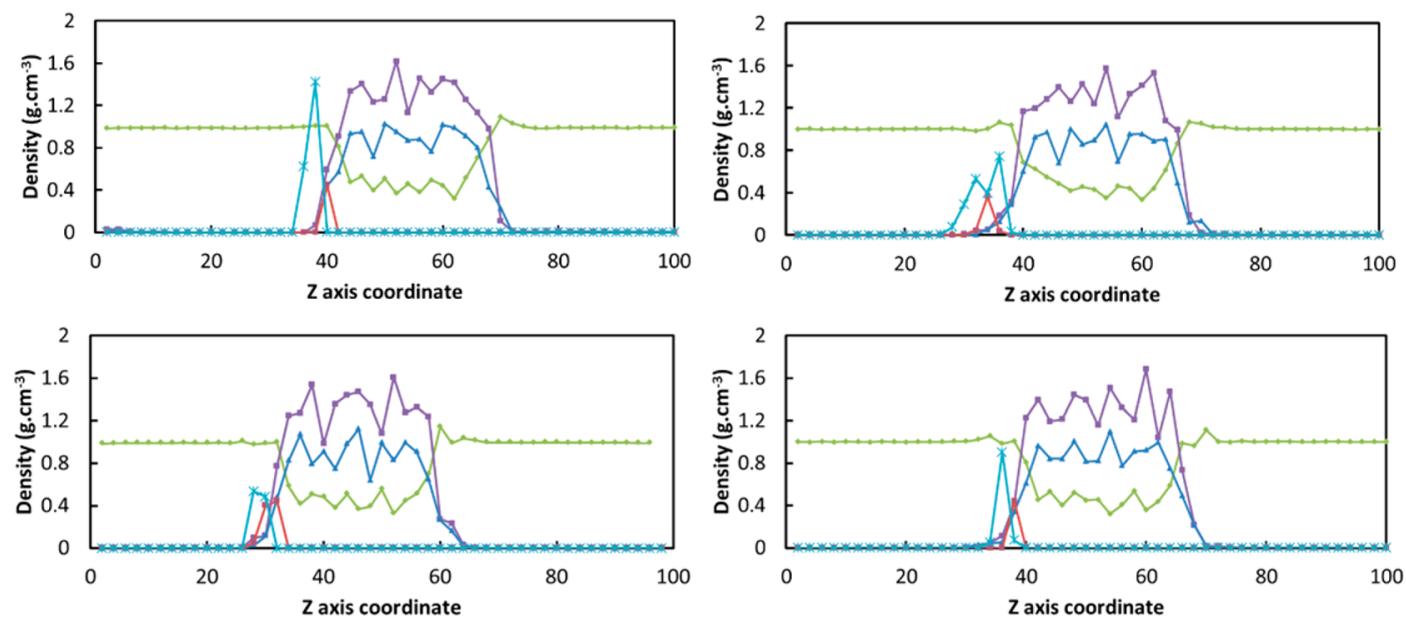

Figure 6. Density profiles of water (green line), carbonate (purple line), calcium (blue line), N (nitrogen in amine or guanidine group - red line), and carboxylate oxygens (light blue line), obtained from $\mathrm{ACC}_{2}$. From left to right and top to bottom: aspartic acid, glutamic acid, arginine, and glycine. The density of the carboxylate oxygens and the nitrogen of the amines was multiplied by 30 to make them visible in the image. 
a

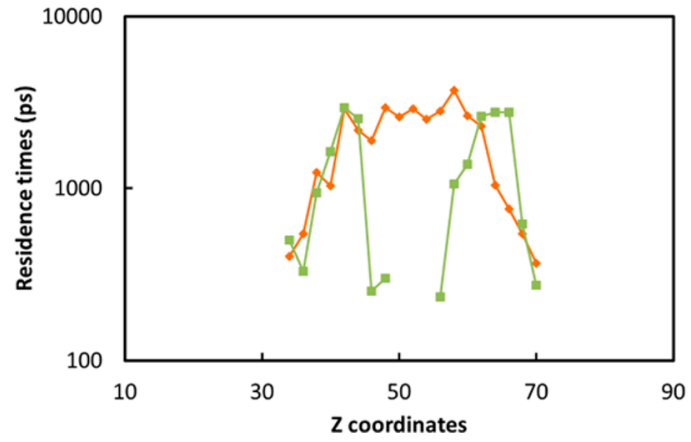

b

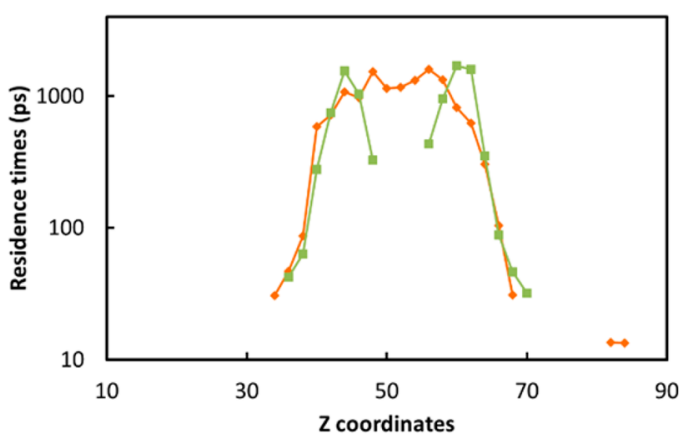

Figure 7. Average times of water molecules within (a) $\mathrm{Ca}^{2+}$ ion and (b) $\mathrm{O}_{\text {carbonate }}$ coordination shell. The green line in the images relates to $\mathrm{ACC}_{1}$ and the orange line to $\mathrm{ACC}_{2}$.
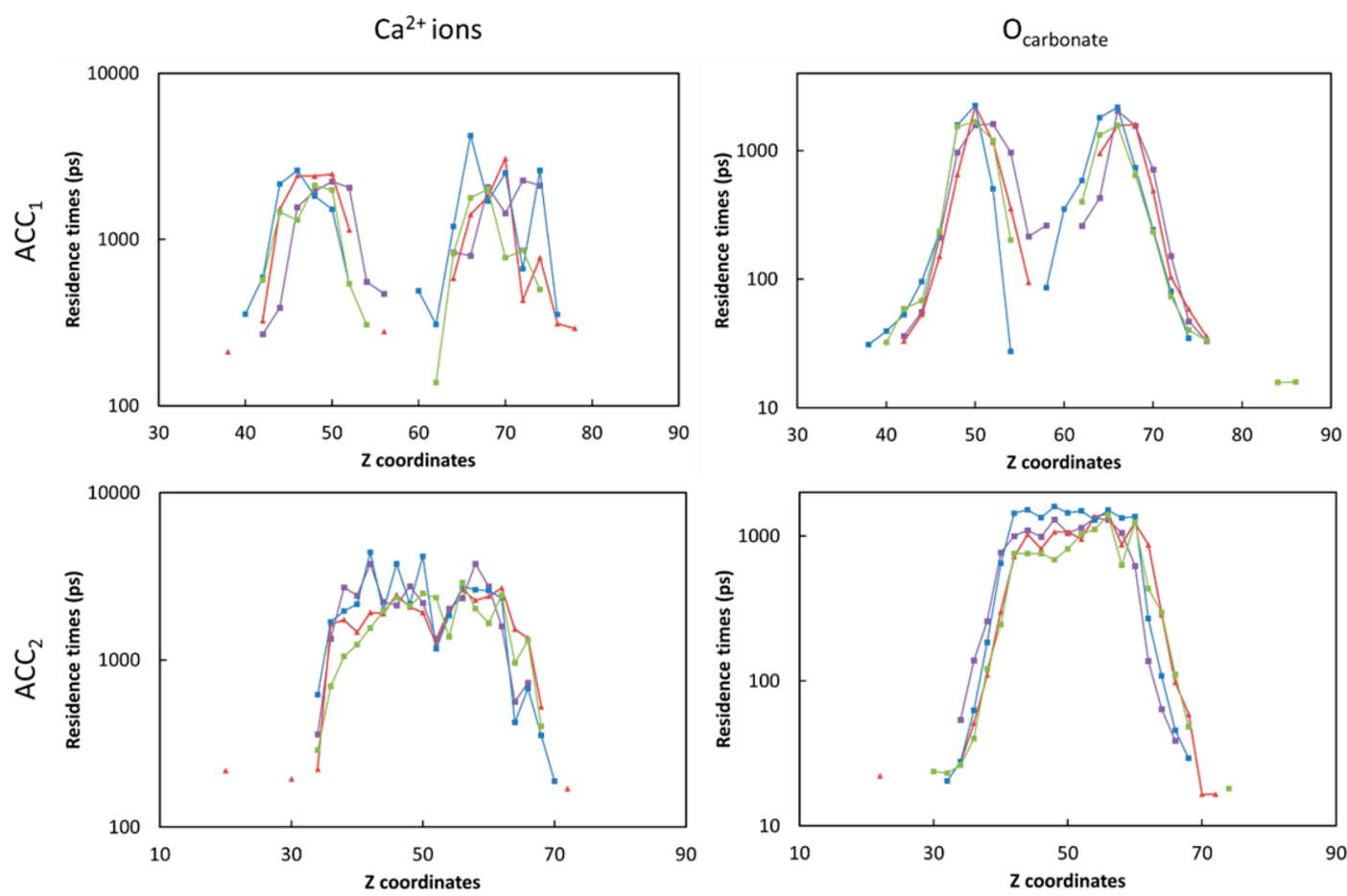

Figure 8. Average times of water molecules within $\mathrm{Ca}^{2+}$ ions and $\mathrm{O}_{\text {carbonate }}$ coordination shell as a function of their position normal to the surface, for both $\mathrm{ACC}_{1}$ and $\mathrm{ACC}_{2}$. The different lines were obtained from simulations containing different amino acids. The green curve was obtained for glutamic acid, the purple one for aspartic acid, the red curve depicts glycine, while the blue one was obtained for arginine.

surroundings, ${ }^{22}$ and thus they might also be able to affect water dynamics.

To demonstrate that the simulation time did not affect the observations presented here, in Figure S16 the average times that water resided in the coordination shell of calcium ions and carbonate oxygens in hydrous and anhydrous ACC are shown for simulations of both 6 and 10 ns. Clearly, increasing the simulation time increases the time that water molecules remain in the shells of atoms in the bulk system. At the surface, however, the calculated times are the same for both simulations, demonstrating that they have reached an equilibrium.

The average time that water molecules stayed in the coordination shells is very similar for $\mathrm{ACC}_{1}$ and $\mathrm{ACC}_{2}$ for both the surface $\mathrm{O}_{\text {carbonates }}$ and $\mathrm{Ca}^{2+}$ ions, as shown in Figure 7. The differences observed in the central bulk region arise due to the lack of water in the center of $\mathrm{ACC}_{1}$. The values found within the first $6 \AA$ of the ACCs' surfaces ranged from 32 to 584 ps and from 272 to $2767 \mathrm{ps}$, for the $\mathrm{O}_{\text {carbonates }}$ and the $\mathrm{Ca}^{2+}$ ions, respectively.
The smallest value found for water molecules in the $\mathrm{O}_{\text {carbonates }}$ coordination shell is larger than the average time for water molecules within the coordination shell of the carbonate ion in bulk solution. This is because $\mathrm{Ca}^{2+}$ ions, present at the ACC surface, impose additional constraints on the dynamics of water molecules, as shown in Figure S17. The values obtained during this study when carbonates $(\sim 14.5 \mathrm{ps})$ and calcium $(170 \mathrm{ps})$ are present in solution are slightly larger than previous $\mathrm{MD}$ simulations (carbonates $-8 \mathrm{ps}$, calcium $87 \mathrm{ps}) .{ }^{51}$ The difference between the calculated values stems from the model used for the calcium carbonate-water interaction. For the potential used in this study, the interactions were fitted to the dissolution free energy of the ions. ${ }^{43}$ Recently, De La Pierre et al. ${ }^{41}$ calculated the residence times of water molecules within the coordination shell of $\mathrm{Ca}^{2+}$ ions in a $\{104\}$ plane of calcite presenting different steps. The values they found ranged from 0.14 to $60 \mathrm{~ns}$, but most values were close to $2 \mathrm{~ns}$. In this case the outermost layers showed values slightly lower on average and were closer to $1 \mathrm{~ns}$, which is 
sensible as the ACC surface is rough and does not structure water as much as calcite, as discussed in the previous sections.

In Figure 8 the average time that water molecules spend within the coordination shell of atoms, depending on their absolute position and for the different amino acids, is presented. The distributions are very similar to those for the ACCs without additives. Amino acids do not seem to have a large impact on the average time that water molecules stay in the coordination shells. This is probably because their effect is localized. It would be complicated to capture exactly how the amino acids modify these average times on a surface as complex as ACC, and to compare the amino acids with one another. As the ACC surface is rough and changes in time, and the amino acids bind to different positions on the surface, examining this issue will require a more focused approach which we leave for future work.

\section{DISCUSSION}

ACC interfaces with water are still poorly defined and experimental studies examining this ubiquitous interface are lacking. The ACCs investigated in this study were shown to have a bulk PDF matching the one obtained experimentally. Analysis of the coordination number of the $\mathrm{Ca}^{2+}$ ions showed a broad distribution. This was divided by separating the calcium ions in different coordination bins. The results showed that two main subpopulations of $\mathrm{Ca}^{2+}$ ions can be defined in bulk $\mathrm{ACC}_{1}$ : one having a coordination number between 7 and 8 and the other having a coordination number between 6 and 7. Similarly, for $\mathrm{ACC}_{2}$, calcium ions either had a coordination number falling between 7 and 8 or a coordination number between 8 and 9. For both ACCs, it was observed that calcium ions with the same coordination tended to cluster together. $\mathrm{Ca}^{2+}$ ion populations with different coordination numbers could explain the change in the ${ }^{13} \mathrm{C}$ NMR shift observed when precipitating a calcium carbonate solution at different $\mathrm{pHs} .{ }^{52}$ Changing the $\mathrm{pH}$ could change their distribution, thereby modifying the NMR shift and potentially influencing polymorph selection.

When the ACC is in contact with water to create a diffuse interface, the ratio between the $\mathrm{Ca}_{6}$ and the $\mathrm{Ca}_{7}$ ions in $\mathrm{ACC}_{1}$ changes from 1.4 to 0.4 . The changes in $\mathrm{ACC}_{2}$ are less significant, but an increase of $\mathrm{Ca}_{8}$ is observed at the surface. It seems that in general the surface of ACC will be populated by $\mathrm{Ca}^{2+}$ ions with a higher number of oxygens in their coordination shell. The smaller size of water molecules allows them to enter into the spaces between carbonate ions, which are not accessible by other carbonate ions. This is clear when looking at $\mathrm{ACC}_{2}$ where the structure is dominated by $\mathrm{Ca}_{7}$ both in bulk and when cut in a slab.

The increase in the number of calcium ions with larger oxygen coordination number is not only due to the presence of water at the surface, but also due to its the penetration into the first $6 \AA$ of the ACC surface. This is most prominent for $\mathrm{ACC}_{1}$ as this surface is initially anhydrous but after $45 \mathrm{~ns}$ of simulation time water penetrated up to $6 \AA$ in depth as also shown by Raiteri and Gale. ${ }^{23}$ This is important as anhydrous ACC may sometimes be considered as completely free of water, which is unlikely to be the case at the interface when in contact with aqueous solutions. Water, however, will diffuse within the surface when in solution, increasing the diffusion of the ions and potentially allowing them to reorganize and slowly form the most stable crystal lattice under the given conditions. This mechanism could explain the quick transformation of anhydrous ACC, when introduced in solution, into one of the stable crystalline structures. ${ }^{4}$ It will be more likely for a crystalline nucleus to form in the surface region, where reconstruction is facilitated by the presence of water molecules.

Analysis of the ACC surface showed that this is diffuse and does not lead to a strong structuring of the water layers ${ }^{22,23}$ as seen in the case of crystalline materials. ${ }^{22,26,47,48}$ Additionally, the results obtained are comparable to previous observations gathered using different force fields (including the one used in the current study) to model the calcium carbonate system. ${ }^{2,23}$ This suggests that the water structure at the interface of ACC is only weakly dependent upon the chosen force field.

All amino acids analyzed showed a favorable free energy of binding to the surface of ACC. This might be a property of the amorphous surface, where the ions can diffuse two orders of magnitude faster than in the bulk solid as mentioned above. The result is that the ionic framework can reorganize to maximize the favorable interactions with the incoming molecules. It could also be that the ACC surface is irregular and presents a number of low energy paths to binding. Previous calculations by Raiteri et al., using nanoparticles of 36 formula units, showed that the barrier to attachment of aspartic acid and acetate was less than $2 \mathrm{~kJ}$ $\mathrm{mol}^{-1}$, which is below thermal energies at room temperature (about $2.5 \mathrm{~kJ} \mathrm{~mol}^{-1}$ ). In good agreement, using a different potential, the largest barrier to attachment found here was of $4 \mathrm{~kJ}$ mol $^{-1}$ observed for glycine on $\mathrm{ACC}_{1}$, while the other molecules did not show any transition barrier between the dissolved and bound states. Comparison of the change of free energy for the binding of aspartic acid found by Raiteri et al. and the current calculation are, however, considerably different. Here a free energy change of $-10.8 \mathrm{~kJ} \mathrm{~mol}^{-1}$ was found, while Raiteri et al. found a value of approximately $-2 \mathrm{~kJ} \mathrm{~mol}^{-1}$. The difference could be ascribed to the force field chosen for the organic molecules, but it could also arise from the difference in the ACC sampled. Raiteri et al. investigated a 36 units nanoparticles and here a surface containing 294 units was analyzed. Additionally, as mentioned in the Methods section, the free energy of binding of aspartic acid to calcium obtained here and in the work of Raiteri et al. is considerably different. Their value ${ }^{24}$ was more exothermic, $-12.2 \mathrm{~kJ} \mathrm{~mol}^{-1}$ compared to the value found here of $-4.5 \mathrm{~kJ} \mathrm{~mol}^{-1}$ (experimental value is $-6.7 \mathrm{~kJ} \mathrm{~mol}^{-1}$ ). ${ }^{37}$ While not explicitly mentioned in their text, the binding could also be different. In this work, it was found that the amino acids predominantly attached to the surface via the amine moiety, while in their work this is not discussed, but it can be assumed that the interaction was through the carboxylate group. It is worth mentioning that for anhydrous nanoparticles of similar sizes to the ones analyzed by Raiteri et al., ${ }^{24}$ Finney and Rodger ${ }^{53}$ found that the surface could be dominated by negatively charged carbonate ions before relaxation in water, which would add a further explanation for the difference found in the free energy of binding. In this work the concentration of the different ions on the surface is similar, as shown by the density plot in Figures 4, 6, S6.

All molecules also showed a favorable change in Helmholtz free energy upon binding. Experimentally, Picker et al. showed that glutamic acid, aspartic acid, and glycine all had an impact on the solubility of ACC, implying that they either interact with the surface or that they modify its structure during precipitation. ${ }^{54}$

The study of Saharay and Kirkpatrick showed that the free energy released from the attachment of calcium carbonate to either guanidine or acetate was approximately the same. ${ }^{25}$ The difference between the two molecules lay in the energy barrier at the transition state. The free energy barrier for the association with acetate was approximately twice as large as the thermal 
energy fluctuations at room temperature, while the one for guanidine was approximately equal. Thus, it is easier for the positively charged guanidine group to interact with ion pairs. A similar result is observed in the present simulations, where all of the amino acids bind through the amine or guanidine groups on both $\mathrm{ACC}_{1}$ and $\mathrm{ACC}_{2}$. Such binding will not be able to occur in peptides and proteins where the basic tails of the amino acids are confined to the backbone of the molecule. Thus, studies using monomeric units of proteins or fragments will not be truly representative of the full molecule. Arginine (and one would expect lysine) is the only exception to this. Its free energy of binding in Figure 5 and the associated snapshots taken at the different minima presented in Figure S3 clearly show that binding through either the guanidine or the amine group is energetically similar. In this case the binding should also represent what happens in proteins if steric hindrances are not considered, and this was observed when analyzing the binding of ovocleidin-17 on amorphous surfaces. ${ }^{22}$

The most favorable binding energy on $\mathrm{ACC}_{1}$ was observed for arginine and glycine, the former being a basic amino acid while the latter is neutral. The former is not usually considered important in biomineralization, as it is found sparingly in protein sequences involved in the process. However, this might be the reason behind its rare observation. As it shows a stronger binding to the surface than the negatively charged amino acids sampled here, it could potentially lock the protein onto the surface and not allow for a subsequent processing step, which might require another organic molecule or another functional domain in the protein itself. Previous simulations of ovocleidin-17 binding on ACC also showed that the attachment is mostly through positively charged residues. They also proposed that the protein could catalyze the transformation of ACC to calcite, then detach from the surface ready for another cycle. This suggests that basic residues may operate as catalyzing agents for ACC conversion to crystalline polymorphs as indicated experimentally, ${ }^{17}$ while acidic residues may not possess the same properties.

On $\mathrm{ACC}_{2}$, the molecules gave a smaller spread of values for the binding energy, and the two strongest binders were glycine and aspartic acid. It seems that the type of the molecule is less important on this hydrous surface. Interestingly, the largest free energy difference in attachment between $\mathrm{ACC}_{1}$ and $\mathrm{ACC}_{2}$ is found for aspartic acid and glutamic acid, while glycine and arginine bind with more or less the same strength to both surfaces. Aspartic and glutamic acid both show a clear preference for $\mathrm{ACC}_{2}$, which can be linked to a decrease in the Coulombic forces. The presence of water will partially screen the charge of the calcium ions in the vicinity of the amino acids as they bind to the carbonate ion. The preference for $\mathrm{ACC}_{2}$ is interesting as it gives clues to the role of these molecules in the stabilization of an amorphous phase. In vitro, Gower et al. showed that polyaspartic acid stabilized a proposed liquid amorphous structure, which has been suggested as a mechanism to form the nonequilibrium morphologies encountered in living systems. ${ }^{5}$ Acidic molecules, are often found in proteins involved in inhibiting mineralization, and these results further confirm their involvement in hydrous ACC, which is often the first to precipitate from solution., ${ }^{5,55}$ Thus, these two acidic molecules could have an important role in stabilizing the hydrous ACC until its morphology is fixed and dehydration can proceed.

Another interesting aspect of the free energy of binding resides in the difference in binding strength between aspartic acid and glutamic acid. On both surfaces analyzed here, aspartic acid binding was associated with a larger change in free energy. This is somewhat counterintuitive as glutamic acid has an additional carbon in its side chain making it more flexible. At a diffuse and fluctuating interface, however, the ions tend to reorganize around the incoming molecule, and, therefore, a longer chain is not necessary to increase the binding strength by interacting with multiple ions and the additional nonpolar carbon may interact unfavorably with the polar environment of the ACC surface.

Analyzing the average times that water molecules stayed in the shells of $\mathrm{Ca}^{2+}$ ions and the $\mathrm{O}_{\text {carbonates }}$ atoms led to some interesting results. At the surface of both the ACCs analyzed, the water molecules appear to behave in a similar way with regard to their diffusion coefficients and the time they spend within the shells of the ions at the surface. This is also true for the averages observed when the amino acids were added to the simulations. This does not mean that the amino acids do not have an impact, but their effect is probably localized, and thus looking at slices through the surface did not highlight it. In general the observed average time the water molecules remained within the coordination shells fell between the value observed in bulk water and the ones observed at the surface of calcite steps. ${ }^{41}$

In conclusion, the results obtained during this study indicate that bulk ACC is composed of regions dominated by calcium ions with different coordination numbers. This effect is clearer in anhydrous ACC and could partially explain the different structures observed experimentally leading to recent ideas about polyamorphism. Additionally, the simulations show that the penetration of water molecules within the ACC surfaces leads to a restructuring of the surface, modifying the coordination shell of the calcium ions by increasing the number of oxygens within it. The surface will thus be at least partially hydrated in aqueous solutions, leading to an increase in the diffusion coefficients of the ions from the center of the slab to the surface. This would facilitate the formation of nuclei for the subsequent crystallization step, but also helps the reorganization of the ions around incoming molecules, simplifying their attachment and partially explaining the favorable change in the free energies upon binding of all the molecules studied here. The attachment occurred predominantly through positively charged groups, (the amine and guanidine moieties), that were hydrogen bonded to carbonate ions. Therefore, studies involving amino acids and/ or short peptides will usually not be representative of larger proteins where the amine groups are involved in the peptide bond. The simulations also showed a clear preference of the negatively charged molecules for hydrated ACC $\left(\mathrm{ACC}_{2}\right)$, which explains their involvement in the inhibition of precipitation and the stabilization of a liquid phase. In future work it would be extremely interesting to investigate in more depth the effect of the organic molecules on the structure of ACC and how this affects the subsequent crystallization.

\section{ASSOCIATED CONTENT}

\section{S Supporting Information}

The Supporting Information is available free of charge on the ACS Publications website at DOI: 10.1021/acs.cgd.7b00874.

The additional material provided includes the free energy curve of binding obtained for the interaction of $\mathrm{C} \mathrm{Ca}^{2+}$ ion with aspartic acid, the position distributions sampled during the umbrella sampling free energy calculations, an example image of the ACC surface, the pair distribution functions of the bulk ACCs analyzed, the $\mathrm{Ca}-\mathrm{O}_{\text {all }}$ coordination numbers of the ACCs, the density profiles of the systems along the $Z$ axis, the configurational energy 
of the analyzed systems as a function of the simulation time, the rate of change of the configurational energy per ns of simulation time, the distribution of the Q6 order parameter of the ACCs in the presence of the amino acids, the $\mathrm{Ca}-\mathrm{O}_{\text {all }}$ coordination numbers of the ACCs in the presence of the amino acids, the distribution of the angle of the carbonate ions with respect to the $x-y$ plane of the ACCs in the presence of the amino acids, the radial distribution function of the ACCs in the presence of the amino acids, the free energy curve of the binding of arginine to $\mathrm{ACC}_{1}$ obtained for different simulation times, the configuration of arginine at the different minima in the free energy curve, a summary of the hydrogen bonds formed between the amino acids and the ACCs surfaces, the distribution of water average residence times of the ACCs for different simulation times, and an image depicting a water molecule within the coordination shell of a $\mathrm{Ca}^{2+}$ and $\mathrm{CO}_{3}{ }^{2-}$ ions. Sample input files can be found in 10.15131/shef.data.5446723 (PDF)

\section{AUTHOR INFORMATION}

\section{Corresponding Author}

*E-mail: riccardo.innocentimalini@empa.ch. Address: Lerchenfeldstrasse 5, 9014 St. Gallen, Switzerland. Phone: 0041587657283.

\section{ORCID $\odot$}

R. Innocenti Malini: 0000-0003-4366-781X

A. R. Finney: 0000-0002-1456-5892

\section{Funding}

Via our membership of the UK's HEC Materials Chemistry Consortium, which is funded by EPSRC (EP/L000202), this work used the ARCHER UK National Supercomputing Service (http://www.archer.ac.uk).

\section{Notes}

The authors declare no competing financial interest.

\section{ACKNOWLEDGMENTS}

All the simulations have been run using the computing facilities provided by the MESAS group at the University of Sheffield. The work was funded by an EPSRC program grant under the MIB project (EP/I001515).

\section{REFERENCES}

(1) Meldrum, F. C.; Colfen, H. Controlling Mineral Morphologies and Structures in Biological and Synthetic Systems. Chem. Rev. 2008, 108, 4332-4432.

(2) Evans, J. S. "Tuning in" to Mollusk Shell Nacre- and PrismaticAssociated Protein Terminal Sequences. Implications for Biomineralization and the Construction of High Performance Inorganic-Organic Composites. Chem. Rev. 2008, 108, 4455-4462.

(3) Mahamid, J.; Aichmayer, B.; Shimoni, E.; Ziblat, R.; Li, C. H.; Siegel, S.; Paris, O.; Fratzl, P.; Weiner, S.; Addadi, L. Mapping amorphous calcium phosphate transformation into crystalline mineral from the cell to the bone in zebrafish fin rays. Proc. Natl. Acad. Sci. U. S. A. 2010, 107, 6316-6321.

(4) Addadi, L.; Raz, S.; Weiner, S. Taking advantage of disorder: Amorphous calcium carbonate and its roles in biomineralization. Adv. Mater. 2003, 15, 959-970.

(5) Gower, L. B.; Odom, D. J. Deposition of calcium carbonate films by a polymer-induced liquid-precursor (PILP) process. J. Cryst. Growth 2000, 210, 719-734.
(6) Beniash, E.; Aizenberg, J.; Addadi, L.; Weiner, S. Amorphous calcium carbonate transforms into calcite during sea urchin larval spicule growth. Proc. R. Soc. London, Ser. B 1997, 264, 461-465.

(7) Falini, G.; Albeck, S.; Weiner, S.; Addadi, L. Control of aragonite or calcite polymorphism by mollusk shell macromolecules. Science 1996, 271, 67-69.

(8) Belcher, A. M.; Wu, X. H.; Christensen, R. J.; Hansma, P. K.; Stucky, G. D.; Morse, D. E. Control of crystal phase switching and orientation by soluble mollusc-shell proteins. Nature 1996, 381, 56-58.

(9) Addadi, L.; Joester, D.; Nudelman, F.; Weiner, S. Mollusk shell formation: A source of new concepts for understanding biomineralization processes. Chem. - Eur. J. 2006, 12, 980-987.

(10) Weiss, I. M.; Tuross, N.; Addadi, L.; Weiner, S. Mollusc larval shell formation: Amorphous calcium carbonate is a precursor phase for aragonite. J. Exp. Zool. 2002, 293, 478-491.

(11) Hasse, B.; Ehrenberg, H.; Marxen, J. C.; Becker, W.; Epple, M. Calcium carbonate modifications in the mineralized shell of the freshwater snail Biomphalaria glabrata. Chem. - Eur. J. 2000, 6, 36793685.

(12) Politi, Y.; Levi-Kalisman, Y.; Raz, S.; Wilt, F.; Addadi, L.; Weiner, S.; Sagi, I. Structural characterization of the transient amorphous calcium carbonate precursor phase in sea urchin embryos. Adv. Funct. Mater. 2006, 16, 1289-1298.

(13) Aizenberg, J.; Lambert, G.; Addadi, L.; Weiner, S. Stabilization of amorphous calcium carbonate by specialized macromolecules in biological and synthetic precipitates. Adv. Mater. 1996, 8, 222.

(14) Olszta, M. J.; Cheng, X. G.; Jee, S. S.; Kumar, R.; Kim, Y. Y.; Kaufman, M. J.; Douglas, E. P.; Gower, L. B. Bone structure and formation: A new perspective. Mater. Sci. Eng., R 2007, 58, 77-116.

(15) Bewernitz, M. A.; Gebauer, D.; Long, J.; Colfen, H.; Gower, L. B. A metastable liquid precursor phase of calcium carbonate and its interactions with polyaspartate. Faraday Discuss. 2012, 159, 291-312.

(16) Levi, Y.; Albeck, S.; Brack, A.; Weiner, S.; Addadi, L. Control over aragonite crystal nucleation and growth: An in vitro study of biomineralization. Chem. - Eur. J. 1998, 4, 389-396.

(17) Cantaert, B.; Kim, Y. Y.; Ludwig, H.; Nudelman, F.; Sommerdijk, N.; Meldrum, F. C. Think Positive: Phase Separation Enables a Positively Charged Additive to Induce Dramatic Changes in Calcium Carbonate Morphology. Adv. Funct. Mater. 2012, 22, 907-915.

(18) Jahromi, M. T.; Yao, G.; Cerruti, M., The importance of amino acid interactions in the crystallization of hydroxyapatite. J. R. Soc., Interface 2013, 10.2012090610.1098/rsif.2012.0906

(19) Freeman, C. L.; Harding, J. H.; Quigley, D.; Rodger, P. M. Structural Control of Crystal Nuclei by an Eggshell Protein. Angew. Chem., Int. Ed. 2010, 49, 5135-5137.

(20) Freeman, C. L.; Harding, J. H.; Quigley, D.; Rodger, P. M. Simulations of Ovocleidin-17 Binding to Calcite Surfaces and Its Implications for Eggshell Formation. J. Phys. Chem. C 2011, 115, 81758183.

(21) Freeman, C. L.; Harding, J. H.; Quigley, D.; Rodger, P. M. Protein binding on stepped calcite surfaces: simulations of ovocleidin-17 on calcite $\{31.16\}$ and $\{31.8\}$. Phys. Chem. Chem. Phys. 2012, 14, 72877295.

(22) Freeman, C. L.; Harding, J. H.; Quigley, D.; Rodger, P. M. How does an amorphous surface influence molecular binding? - ovocleidin-17 and amorphous calcium carbonate. Phys. Chem. Chem. Phys. 2015, 17, 17494-17500.

(23) Raiteri, P.; Gale, J. D. Water Is the Key to Nonclassical Nucleation of Amorphous Calcium Carbonate. J. Am. Chem. Soc. 2010, 132, 1762317634.

(24) Raiteri, P.; Demichelis, R.; Gale, J. D.; Kellermeier, M.; Gebauer, D.; Quigley, D.; Wright, L. B.; Walsh, T. R. Exploring the influence of organic species on pre- and post-nucleation calcium carbonate. Faraday Discuss. 2012, 159, 61-85.

(25) Saharay, M.; Kirkpatrick, R. J. Ab initio and metadynamics studies on the role of essential functional groups in biomineralization of calcium carbonate and environmental situations. Phys. Chem. Chem. Phys. 2014, 16, 26843-26854. 
(26) Fenter, P.; Kerisit, S.; Raiteri, P.; Gale, J. D. Is the Calcite-Water Interface Understood? Direct Comparisons of Molecular Dynamics Simulations with Specular X-ray Reflectivity Data. J. Phys. Chem. C 2013, 117, 5028-5042.

(27) Malini, R. I.; Bushuev, Y. G.; Hall, S. A.; Freeman, C. L.; Rodger, P. M.; Harding, J. H. Using simulation to understand the structure and properties of hydrated amorphous calcium carbonate. CrystEngComm 2016, 18, 92-101.

(28) Radha, A. V.; Forbes, T. Z.; Killian, C. E.; Gilbert, P. U. P. A.; Navrotsky, A. Transformation and crystallization energetics of synthetic and biogenic amorphous calcium carbonate. Proc. Natl. Acad. Sci. U. S. A. 2010, 107, 16438-16443.

(29) Martinez, L.; Andrade, R.; Birgin, E. G.; Martinez, J. M. PACKMOL: A Package for Building Initial Configurations for Molecular Dynamics Simulations. J. Comput. Chem. 2009, 30, 21572164.

(30) Todorov, I. T.; Smith, W.; Trachenko, K.; Dove, M. T. DL_POLY_3: new dimensions in molecular dynamics simulations via massive parallelism. J. Mater. Chem. 2006, 16, 1911-1918.

(31) Wu, Y.J.; Tepper, H. L.; Voth, G. A., Flexible simple point-charge water model with improved liquid-state properties. J. Chem. Phys. 2006, $124.02450310 .1063 / 1.2136877$

(32) Hornak, V.; Abel, R; Okur, A.; Strockbine, B.; Roitberg, A.; Simmerling, C. Comparison of multiple AMBER force fields and development of improved protein backbone parameters. Proteins: Struct., Funct., Genet. 2006, 65, 712-725.

(33) Kahlen, J.; Salimi, L.; Sulpizi, M.; Peter, C.; Donadio, D. Interaction of Charged Amino-Acid Side Chains with Ions: An Optimization Strategy for Classical Force Fields. J. Phys. Chem. B 2014, 118, 3960-3972.

(34) Sparks, D. J.; Romero-Gonzalez, M. E.; El-Taboni, E.; Freeman, C. L.; Hall, S. A.; Kakonyi, G.; Swanson, L.; Banwart, S. A.; Harding, J. H. Adsorption of poly acrylic acid onto the surface of calcite: an experimental and simulation study. Phys. Chem. Chem. Phys. 2015, 17, 27357-27365.

(35) Cote, A. S.; Darkins, R.; Duffy, D. M. Deformation twinning and the role of amino acids and magnesium in calcite hardness from molecular simulation. Phys. Chem. Chem. Phys. 2015, 17, 20178-20184.

(36) Freeman, C. L.; Harding, J. H.; Cooke, D. J.; Elliott, J. A.; Lardge, J. S.; Duffy, D. M. New forcefields for modeling biomineralization processes. J. Phys. Chem. C 2007, 111, 11943-11951.

(37) Covington, A. K.; Danish, E. Y. Measurement of magnesium stability constants of biologically relevant ligands by simultaneous use of $\mathrm{pH}$ and ion-selective electrodes. J. Solution Chem. 2009, 38, 1449-1462.

(38) Hamm, I. M.; Wallace, A. F.; Dove, P. M. Molecular Dynamics of ion hydration in the presence of small carboxylated molecules and implications for calcification. J. Phys. Chem. B 2010, 114, 10488-10495.

(39) Bonomi, M.; Branduardi, D.; Bussi, G.; Camilloni, C.; Provasi, D.; Raiteri, P.; Donadio, D.; Marinelli, F.; Pietrucci, F.; Broglia, R. A.; Parrinello, M. PLUMED: A portable plugin for free-energy calculations with molecular dynamics. Comput. Phys. Commun. 2009, 180, 19611972.

(40) Grossfield, A. WHAM: the weighted histogram analysis method. http://membrane.urmc.rochester.edu/content/wham.

(41) De La Pierre, M.; Raiteri, P.; Gale, D. J. Structure and dynamics of water at step edges on the calcite $\{104\}$ surface. Cryst. Growth Des. 2016, 16, 5907-5914.

(42) Pavese, A.; Catti, M.; Parker, S. C.; Wall, A. Modelling of the thermal dependence of structural and elastic properties of calcite, CaCO3. Phys. Chem. Miner. 1996, 23, 89-93.

(43) Raiteri, P.; Gale, J. D.; Quigley, D.; Rodger, P. M. Derivation of an Accurate Force-Field for Simulating the Growth of Calcium Carbonate from Aqueous Solution: A New Model for the Calcite-Water Interface. J. Phys. Chem. C 2010, 114, 5997-6010.

(44) Bano, A. M.; Rodger, P. M.; Quigley, D. New Insight into the Stability of $\mathrm{CaCO} 3$ Surfaces and Nanoparticles via Molecular Simulation. Langmuir 2014, 30, 7513-7521.
(45) Bushuev, Y. G.; Finney, A. R.; Rodger, P. M. Stability and Structure of Hydrated Amorphous Calcium Carbonate. Cryst. Growth Des. 2015, 15, 5269-5279.

(46) Raiteri, P.; Demichelis, R.; Gale, J. D. Thermodynamically Consistent Force Field for Molecular Dynamics Simulations of AlkalineEarth Carbonates and Their Aqueous Speciation. J. Phys. Chem. C 2015, 119, 24447-24458.

(47) Spagnoli, D.; Kerisit, S.; Parker, S. C. Atomistic simulation of the free energies of dissolution of ions from flat and stepped calcite surfaces. J. Cryst. Growth 2006, 294, 103-110.

(48) Kerisit, S.; Cooke, D. J.; Spagnoli, D.; Parker, S. C. Molecular dynamics simulations of the interactions between water and inorganic solids. J. Mater. Chem. 2005, 15, 1454-1462.

(49) Lahav, N.; Bolt, G. H. Self-diffusion of Ca into certain carbonates. Soil Sci. 1964, 97, 293-299.

(50) Dorvee, J. R.; Veis, A. Water in the formation of biogenic minerals: Peeling away the hydration layers. J. Struct. Biol. 2013, 183, 278-303.

(51) Kerisit, S.; Liu, C. X. Molecular simulation of the diffusion of uranyl carbonate species in aqueous solution. Geochim. Cosmochim. Acta 2010, 74, 4937-4952.

(52) Gebauer, D.; Gunawidjaja, P. N.; Ko, J. Y. P.; Bacsik, Z.; Aziz, B.; Liu, L. J.; Hu, Y. F.; Bergstrom, L.; Tai, C. W.; Sham, T. K.; Eden, M.; Hedin, N. Proto-Calcite and Proto-Vaterite in Amorphous Calcium Carbonates. Angew. Chem., Int. Ed. 2010, 49, 8889-8891.

(53) Finney, A. R.; Rodger, P. M. Probing the structure and stability of calcium carbonate pre-nucleation clusters. Faraday Discuss. 2012, 159, $47-60$.

(54) Picker, A.; Kellermeier, M.; Seto, J.; Gebauer, D.; Colfen, H. The multiple effects of amino acids on the early stages of calcium carbonate crystallization. Z. Kristallogr. - Cryst. Mater. 2012, 227, 744-757.

(55) Cheng, X. G.; Varona, P. L.; Olszta, M. J.; Gower, L. B. Biomimetic synthesis of calcite films by a polymer-induced liquidprecursor (PILP) process 1. Influence and incorporation of magnesium. J. Cryst. Growth 2007, 307, 395-404. 